\title{
Three-dimensional crystallographic characterization and mechanical modeling of a commercial stainless steel
}

\author{
A. C. Lewis, D. J. Rowenhorst, G. Spanos \& A. B. Geltmacher \\ Multifunctional Materials Branch, U. S. Naval Research Laboratory, \\ Washington, DC, USA
}

\begin{abstract}
The microstructure, crystallography, and mechanical response of a commercial super-austenitic stainless steel, AL-6XN, has been investigated. Three-dimensional (3D) reconstructions, generated by combining serial sectioning techniques with Electron Backscatter Diffraction (EBSD), are used for characterization of the microstructure and crystallography of the material, and as input for 3D Image-Based Finite Element Models (IB-FEM). Using these techniques, the distributions of crystallographic 3D grain boundary normals have been quantified, and are shown to provide critical new information not previously attainable from the commonly used morphological descriptors. Image-based finite element simulations, with the 3D microstructure and crystallography as input, were performed to determine the critical microstructural features at which failure is likely to initiate.
\end{abstract}

Keywords: $3 D$ microstructures, $3 D$ analysis, austenitic stainless steel, crystallography, finite element modeling.

\section{Introduction}

Three-dimensional experimental materials characterization and analyses are becoming increasingly prevalent, as recent advances have made both the acquisition of 3D datasets and the analysis of these data faster and more readily available (i.e. [1-4]). As material data evolves from classic two-dimensional micrographs and cross-sectional images to complete three-dimensional datasets, the modeling and simulation techniques used to analyze the properties and predict the performance of these materials must also adapt to accommodate these 
3D microstructures. Particularly in the case of mechanical performance, the true 3D microstructural characteristics of a material must be understood in order to model its behavior accurately [5-11]. In addition, crystallographic orientation, which plays a critical role in the elastic and plastic behavior of many materials, must be considered in the simulation of mechanical behavior at the mesoscale. With complete 3D spatial and crystallographic information of a given microstructure, Image-Based Finite Element Modeling (IB-FEM) can be used to determine critical microstructural features where failure or fracture is likely to initiate in the material.

To understand fully the crystallography of a material, it is essential to use three-dimensional information about the grain morphology and orientation, particularly when studying grain boundaries. While a great deal of information on grain orientation, boundary structure, and misorientation can be obtained from conventional electron backscatter diffraction (EBSD) techniques, the complete (5-parameter) description of a grain boundary cannot be sufficiently determined from extrapolations of 2D data [12]. Combining experimentallydetermined spatial and crystallographic microstructural data and using these data as input for finite element modelling of mechanical response thus provides a wealth of information not previously available for real $3 \mathrm{D}$ microstructures, and through the quantitative analysis of these data, a complete characterization of material microstructures can be established.

\section{Materials and methods}

The material examined in this study is AL-6XN, a commercially available superaustenitic stainless steel. The as-received material was in the form of a continuously cast and milled annealed $\left(>1100^{\circ} \mathrm{C}\right) 6.35 \mathrm{~mm}$-thick $(1 / 4$ ") plate. The mechanical properties of AL-6XN have been reported in detail [13], including high observed ductility (strain $>40 \%$ ) over a broad range of temperatures, and little sensitivity to strain rate. A second phase $(\sigma)$ has been observed at the centerline of AL-6XN plates, which can result in reduction in mechanical performance and initiation of fracture and failure [14]. This work focuses on the austenite matrix, and specimens were collected far from the centerline to avoid any interaction of the austenite with the second phase precipitates.

Serial sectioning in conjunction with optical microscopy and automated EBSD were used to create a three-dimensional reconstruction of the austenite matrix, incorporating crystallographic orientation of austenite grains. Optical micrographs were recorded for each section, followed by the removal of approximately $3.3 \mu \mathrm{m}$ of material by mechanical polishing. This process was repeated over 100 times, with EBSD scans recorded every tenth section (every $33 \mu \mathrm{m})$. Image processing was performed using Matlab and Photoshop software packages, and 3D reconstruction and data analyses were performed using Matlab and the Interactive Data Language (IDL). Further details of the data collection and 3D reconstruction procedures are provided in prior works [15], in which the same techniques were applied to a preliminary dataset. 
A complete set of 3D microstructural analyses was applied to the reconstructed volume. The dataset consists of a 3D array of integers, each representing a grain identification number, and corresponding to each grain ID there is an associated crystallographic orientation, averaged over all measured values for that grain. Formatting the data in this way make microstructural characterization relatively straightforward - grain volumes, for example, are calculated by recording the number of voxels in each grain, and multiplying by the appropriate microns-per-voxel conversion. Grain aspect ratios are measured by fitting a 3D ellipsoid to each individual grain and twin, and calculating the major axis and the shortest minor axis. This measure, along with the number of facets each grain has, gives a quantitative measure of the grain morphology, without the need for categorizing the varied and complex shapes found within such a highly twinned microstructure.

A single crystallographic orientation was assigned to each grain and twin within the 3D microstructure, based on the averaged values measured using EBSD. Attaining the true 3D shape of each grain and twin along with its crystallography allows for the measurement of the complete 5-parameter grain boundary definition $[12,16]$. Although this dataset contains just 120 complete reconstructed grains, the number of interfaces between grains number over 2000, and it is not necessary to reconstruct the entire surface between two grains to determine the misorientation between them, or, in most cases, the complete grain boundary definition. The convention used here to describe the grain boundary plane is to define the two crystallographic normals of the intersecting plane (with respect to the two grains on either side of the plane), and the angle of twist between them.

In addition to microstructural and crystallographic characterization, the simulated mechanical response of this material was studied using image-based finite element modeling, with the 3D spatial and crystallographic reconstruction as input. The finite element mesh is a regular rectangular grid generated using the 3D microstructural data array, and defining one element per voxel. Due to constraints on file size and computation time, the mesh for these simulations was created by sampling every fourth voxel in each sectioning $(x-y)$ plane. Each element is therefore $3.6 \times 3.6 \times 3.3 \mu \mathrm{m}$ in size and comprises the ABAQUS ${ }^{\circledR}$ C3D8 element type. Each of the 346 grains is represented by an individual element set, and each element set is assigned the average calculated crystallographic orientation for that grain. All simulations were run using anisotropic elasticity. Further details of the meshing and simulation techniques were reported previously for work on a smaller, preliminary data set [10].

\section{3D microstructural analysis}

A $3 \mathrm{D}$ reconstruction of the AL-6XN microstructure is shown in Figure 1. The reconstructed volume measures $450 \times 450 \times 260 \mu \mathrm{m}$, and contains 346 grains and twins. (For the purposes of this analysis, a "grain" is defined by a single orientation, and twins are therefore considered as separate from their parent grains.) Of these 346 reconstructed grains, 120 are completely contained within the dataset, that is, they do not intersect the edges of the reconstructed volume. 


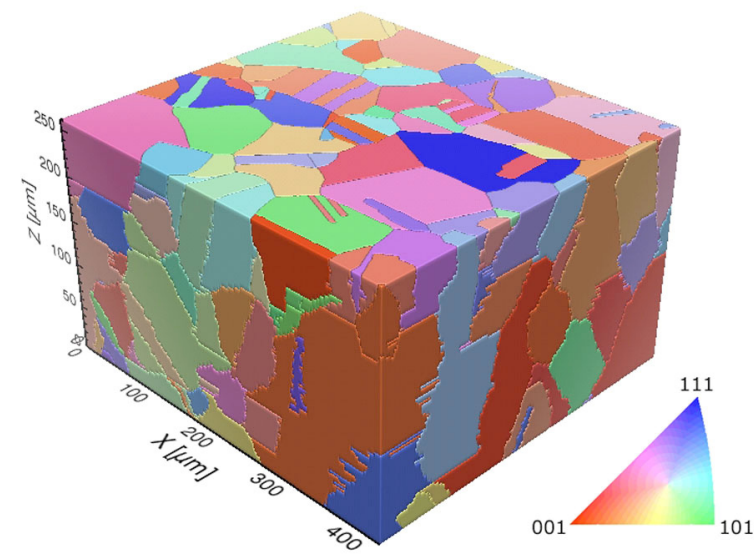

Figure 1: $3 \mathrm{D}$ reconstruction of the austenite phase of AL-6XN. The color of each individual grain and twin corresponds to the crystallographic orientation parallel to the sample $z$-axis, according to the legend.

Two- and three-dimensional analyses of the AL-6XN microstructure were performed on a preliminary dataset, reported previously [15]. The preliminary dataset, however, contained only a handful of "interior" grains not intersecting the edges of the reconstructed volume. The current dataset shown in Figure 1 contains 120 such grains, and although this does not represent a statistically significant number of grains, analysis of the size and shape of these grains does provide useful information with respect to the microstructural characterization of this material.

Figure 2 shows the grain size distribution for the 120 interior grains in the above microstructure. The values reported are the spherical equivalent radius, calculated based on the volume of each individual grain, which is equal to the number of voxels each grain comprises, multiplied by the appropriate cubic micron-per-voxel conversion (in this dataset, one voxel equals approximately 2.7 $\mu \mathrm{m}^{3}$ ). The average (mean) measured grain volume is $156,000 \mu \mathrm{m}^{3}$, or approximately $(54 \mu \mathrm{m})^{3}$. Figure 3 shows the distribution of 3D grain aspect ratios for the interior grains in the microstructure. The grain aspect ratio is measured by fitting an ellipsoid to each grain, and measuring the major axis, divided by the shortest minor axis. Note that the majority of the grains in this microstructure have an aspect ratio much greater than 1, suggesting that there are very few equiaxed grains, and that the distribution of shapes in this microstructure is quite complex. Previous 2D measurements of the crystallography of this material have shown that the microstructure contains a significant number of recrystallization twins with the $\Sigma=3$ coincident site lattice (CSL) misorientation relationship [15]. Visualization of the reconstructed 3D microstructure confirms that a large number of these twins have plate-like morphologies, and therefore fall into the larger end of the aspect ratio distribution. 


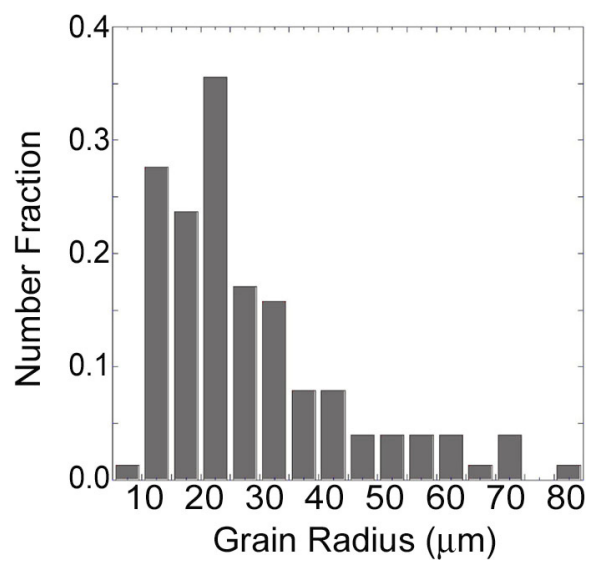

Figure 2: Grain size distribution for the "interior" grains in the reconstructed 3D microstructure shown in Figure 1.

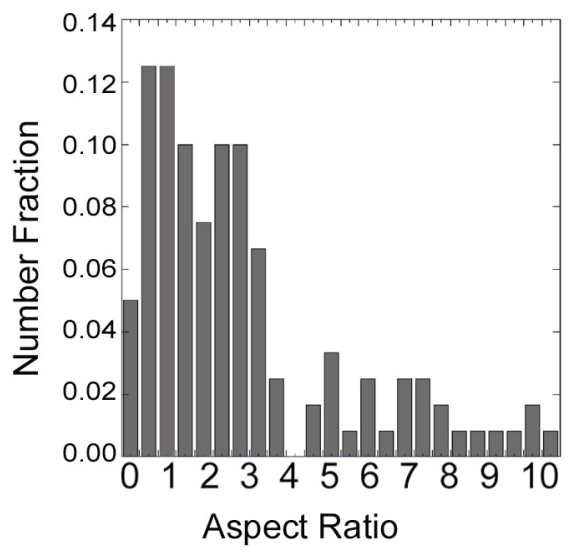

Figure 3: 3D aspect ratio distribution for the "interior" grains in the reconstructed 3D microstructure shown in Figure 1.

Figure 4 shows 3D reconstructions of the grain boundary networks in this AL-6XN volume. In this figure, grain boundaries and junctions are color-coded based on the junction type. Only two types of boundary surfaces were considered here: $\Sigma 3 \mathrm{CSL}$ boundaries (light color in the figure), and general high-angle $\left(>15^{0}\right)$ grain boundaries (dark color in the figure). The triple junction networks were also reconstructed, but are not shown in the figure. The $3 \mathrm{D}$ reconstruction of the boundary and junction networks in this microstructure, particularly the network of $\Sigma 3$ twin boundaries, are used for additional analysis of crystallography, corrosion properties, and, using image-based FEM, the critical microstructural features where plasticity is likely to initiate. 


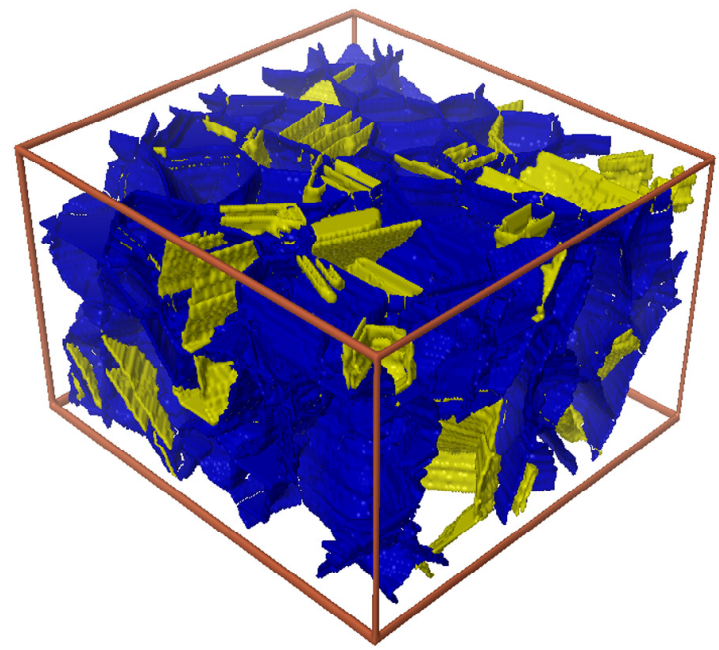

Figure 4: 3D reconstruction of grain boundaries in the AL-6XN microstructure shown in Figure 1. General high-angle grain boundaries are shown in blue (dark color), $\Sigma 3$ CSL boundaries are shown in yellow (light color).

\section{Crystallographic analysis}

Because this microstructure is so highly twinned, a wide range of grain sizes and shapes are present, and conventional morphological descriptors are not sufficient to characterize it completely; the crystallography must therefore also be quantified. Using the information obtained from serial sectioning and EBSD, the crystallographic normals of each grain boundary plane can be determined, and boundaries between two grains can be defined completely by the two crystallographic normals of the intersecting plane (with respect to the two grains on either side of the plane), and the angle of twist between them [15].

To quantify the crystallography in the AL-6XN microstructure, the crystallographic normals were calculated for all boundary surfaces. Rather than fit a plane to each grain boundary and determine a single set of normal vectors, the microstructure was converted to a 3D surface mesh, and the crystallographic normal for each patch or "sub-surface" on the mesh was calculated and plotted. (Details of this procedure are reported elsewhere [2].) Figure 5 is a Crystallographic Interface Normal Distribution (CIND) plot for all surfaces within the AL-6XN microstructure shown in Figure 1.

In the figure, the largest peaks are observed near the $<101>$ and $<113>$ directions. The largest concentration of normal directions is within $5^{0}$ of the $<101>$ directions. This peak corresponds to $<101>\Sigma 3$ twist boundaries, which dominate the twin structure. Note that, although it is the lowest energy family of boundary planes, the strongest peak is not found near the $<111>$ normal direction. 


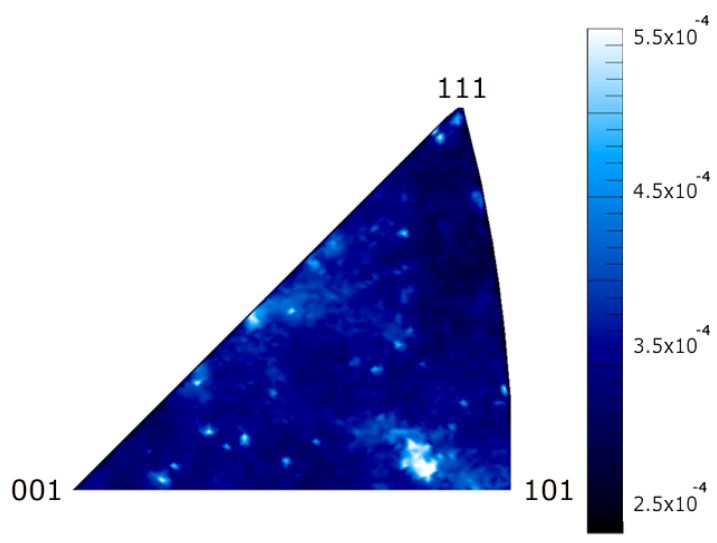

Figure 5: Crystallographic Interface Normal Distribution (CIND) plot for each interface in the AL-6XN microstructure shown in Figure 1.

\section{Image-based finite element modeling}

The 3D spatial and crystallographic information obtained from serial sectioning and EBSD was used as input into an image-based finite element model, to simulate the response of the microstructure to a variety of loads under a number of different constraints. The model was tested under uniaxial stretch, plane strain tension and uniaxial tension conditions by applying the appropriate displacement boundary conditions to the faces of the model. A detailed quantitative analysis of the simulated mechanical response was performed, and is to be reported a future manuscript [17]; a few examples of the type of analysis and visualization techniques are presented here.

Figure 6 is a contour plot of the von Mises stress as a result of $0.2 \%$ applied elastic strain in the $x$-direction, as indicated by the axes in the figure. In this simulation, the strain was applied uniformly to the positive $x$-face, and all other faces were constrained to be fixed. Variations in von Mises stress resulting from this strain due to local anisotropy are visible in the figure.

In these datasets, quantitative data mining and advanced scientific visualization techniques are used to determine correlations between microstructure and mechanical properties. In previous studies [1, 10], high stresses have been observed at grain boundaries and junctions. In particular, the behavior of $\Sigma 3$ boundaries under different loading conditions is of interest, as it has been observed that the preferential response of these boundaries depends on the loading conditions applied [17].

To determine the effect of crystallography and grain structure on mechanical behavior, the mechanical response of the material around grain boundaries and triple junctions was studied under a variety of loading conditions. Finite element simulation outputs such as von Mises stress and maximum principal strain for the reconstructed volume were analyzed at specific crystallographic and microstructural features, including general and special grain boundaries and 
triple junctions. It was found that, under constrained uniaxial tensile loading, values of von Mises stress are highest near the junctions between two $\Sigma 3$ grain boundaries. This is not the case, however, for simple shear loading, which results in highly variable stresses at these junctions, which in some cases are lower than at any other junction type.

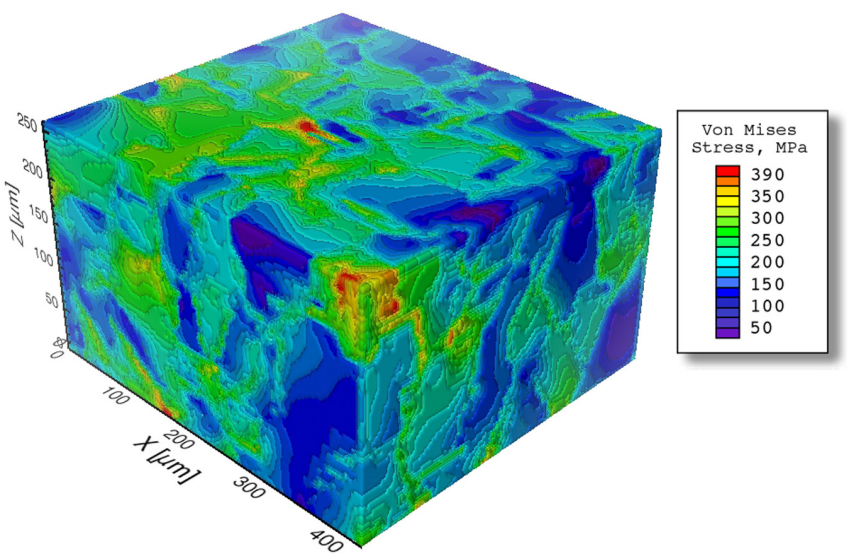

Figure 6: Contour plot of von Mises stress resulting from $0.2 \%$ applied elastic strain in the $x$-direction.

\section{Conclusions}

The microstructure, crystallography, and mechanical response of a commercial super-austenitic stainless steel, AL-6XN, was investigated. Three-dimensional reconstructions, generated by combining serial sectioning techniques with (EBSD), are used for characterization of the microstructure and crystallography of the material, and as input for 3D Image-Based Finite Element Models. Using these techniques, the distributions of crystallographic 3D grain boundary normals have been quantified, and are shown to provide critical new information not previously attainable. The microstructure, which is dominated by $\Sigma 3$ recrystallization twins, contains a majority of $<101>$ twist boundaries. Imagebased finite element simulations, with the 3D microstructure and crystallography as input, were performed to determine the critical microstructural features at which failure is likely to initiate. This combination of 3D crystallography, morphology, and mechanical modeling provides new insight into the characterization and analysis of this material not possible with 2D imaging and simulations.

\section{Acknowledgements}

This work was jointly sponsored by the Office of Naval Research (ONR) and DARPA as part of the Dynamic 3-D ("D3D") Digital Structures program. Additional funding was from ONR under the "Design of Naval Steels" program. 
The authors wish to express their gratitude to Mr. Leroy Levenberry, Ms. Jessica Woods and Mr. Jehud Flores for their significant efforts in the serial sectioning and image processing of the data. The material used in this study was provided by Mr. E. Czyryca at the Naval Surface Warfare Center - Carderock Division.

\section{References}

[1] Lewis, A.C., et al., Quantitative analysis and feature recognition in 3D microstructural data sets. JOM, 58(12), p 52-56, 2006.

[2] Rowenhorst, D.J., et al., 3D crystallographic and morphological analysis of coarse martensite: Combining EBSD and serial sectioning. Scripta Materialia, 55(1): p. 11-16, 2006.

[3] Spowart, J.E., Automated serial sectioning for 3-D analysis of microstructures. Scripta Materialia, 55(1): p. 5-10, 2006.

[4] Uchic, M.D., et al., 3D microstructural characterization of nickel superalloys via serial-sectioning using a dual beam FIB-SEM. Scripta Materialia, 55(1): p. 23-28, 2006.

[5] Cailletaud, G., et al., Some elements of microstructural mechanics. Computational Materials Science, 27(3): p. 351-374, 2003.

[6] Chawla, N. and Deng, X., Microstructure and mechanical behavior of porous sintered steels. Materials Science and Engineering A, 390(1-2): p. 98-112, 2005.

[7] Chawla, N., Ganesh, V.V., and Wunsch, B., Three-dimensional (3D) microstructure visualization and finite element modeling of the mechanical behavior of $\mathrm{SiC}$ particle reinforced aluminum composites. Scripta Materialia, 51(2): p. 161-165, 2004.

[8] Ghosh, S. and Moorthy, S., Three dimensional Voronoi cell finite element model for microstructures with ellipsoidal heterogeneties. Computational Mechanics, 34(6): p. 510-531, 2004.

[9] Kenesei, P., Borbely, A., and Biermann, H., Microstructure based threedimensional finite element modeling of particulate reinforced metalmatrix composites. Materials Science and Engineering A, 387-89: p. 852856, 2004.

[10] Lewis, A.C. and Geltmacher, A.B., Image-based modeling of the response of experimental 3D microstructures to mechanical loading. Scripta Materialia, 55(1): p. 81-85, 2006.

[11] Youssef, S., Maire, E., and Gaertner, R., Finite element modelling of the actual structure of cellular materials determined by X-ray tomography. Acta Materialia, 53: p. 719-730, 2005.

[12] Wolf, D. and Yip, S., eds. Materials Interfaces: Atomic-level structure and properties. 1992, Chapman and Hall: London.

[13] Nemat-Nasser, S., Guo, W.G., and Kihl, D.P., Thermomechanical response of AL-6XN stainless steel over a wide range of strain rates and temperatures. Journal of the Mechanics and Physics of Solids, 49(8): p. 1823-1846, 2001. 
124 Computational Methods and Experiments in Materials Characterisation III

[14] Stauffer, A.C., Koss, D.A., and McKirgan, J.B., Microstructural banding and failure of a stainless steel. Metallurgical and Materials Transactions A, 35A(4): p. 1317-1324, 2004.

[15] Lewis, A.C., et al., Two- and three-dimensional microstructural characterization of a super-austenitic stainless steel. Materials Science and Engineering A, 418(1-2): p. 11-18, 2006.

[16] Randle, V. and Engler, O., Introduction to Texture Analysis: Macrotexture, Microtexture and Orientation Mapping, CRC Press, Boca Raton, FL, 2000.

[17] Lewis, A.C., Geltmacher, A.B., and Jordan, K.A.: p. (unpublished research). 2007. 